\title{
Impaired psychomotor ability and attention in patients with persistent pain: a cross-sectional comparative study
}

This article was published in the following Dove Press journal:

Journal of Pain Research

17 October 2016

Number of times this article has been viewed

\author{
Helena Gunnarsson ${ }^{1,2}$ \\ Birgitta Grahn ${ }^{3-5}$ \\ Jens Agerström' \\ 'Department of Psychology, Faculty \\ of Health and Life Sciences, Linnaeus \\ University, Vaxjo, ${ }^{2} \mathrm{Hälsoringen,} \mathrm{Neron}$ \\ HSU AB, Osby, ${ }^{3}$ Department of \\ Clinical Sciences Lund-Orthopedics, \\ Lund University, ${ }^{4}$ Epidemiology and \\ Register Centre South, Region Skåne, \\ Lund, ${ }^{5}$ Department of Research and \\ Development, Region Kronoberg, \\ Vaxjo, Sweden
}

Background and aims: Patients with pain have shown cognitive impairment across various domains. Although the pain qualities vary among patients, research has overlooked how cognitive performance is affected by the duration and persistence of pain. The current study sought to fill this gap by examining how qualitatively different pain states relate to the following cognitive functions: sustained attention, cognitive control, and psychomotor ability.

Patients and methods: Patients with musculoskeletal pain in primary care were divided into three pain groups: acute pain (duration $<3$ months), regularly recurrent pain (duration $>3$ months), and persistent pain (duration $>3$ months). These groups were then compared with healthy controls. The MapCog Spectra Test, the Color Word Test, and the Grooved Pegboard Test were used to measure sustained attention, cognitive control, and psychomotor ability, respectively.

Results: Patients with persistent pain showed significantly worse sustained attention and psychomotor ability compared with healthy controls. The acute pain group showed a significant decrease in psychomotor ability, and the regularly recurrent pain group showed a significant decrease in sustained attention. These results remained unchanged when age, education, and medication were taken into account.

Conclusion: Persistent musculoskeletal pain seems to impair performance on a wider range of cognitive tasks than acute or regularly recurrent pain, using pain-free individuals as a benchmark. However, there is some evidence of impairment in psychomotor ability among patients with acute pain and some impairment in sustained attention among patients with regularly recurrent pain.

Implications: Caregivers may need to adjust communication methods when delivering information to cognitively impaired patients.

Keywords: persistent pain, cognitive impairment, musculoskeletal pain, psychomotor ability, attention, cognitive control

\section{Introduction}

Compared to pain-free controls, patients with pain have been found to perform worse on cognitive outcomes such as memory, attention, speed in performing structured tasks, psychomotor ability, verbal ability, number sense, and mental flexibility; ${ }^{1-9}$ however, studies have been inconsistent. ${ }^{10-20}$ Although there seems to be a growing interest in the relationship between pain and cognition, researchers have largely overlooked how cognitive performance is affected by different characteristics of pain apart from its intensity. This is noteworthy given that the type of pain people suffer from can vary on many other dimensions than intensity. The current study sought to fill this research
Correspondence: Helena Gunnarsson Department of Psychology, Faculty of Health and Life Sciences, Linnaeus University, University Place I, 35195 Vaxjo, Sweden

$\mathrm{Tel}+46772288000$

Fax +4647083217

Email helena.gunnarsson@Inu.se 
void by examining how pain duration and pain persistence relate to sustained attention, cognitive control, and psychomotor ability.

The notion that the duration and the persistence of pain should matter for cognitive performance is supported by a three-part model and an associated research on pain-related cognitive impairment, brain imaging, and brain neurochemistry. ${ }^{10}$ According to the limited resource part, pain signals from the spinal cord to brain regions (eg, the prefrontal cortex, the anterior cingulate cortex, and the secondary somatosensory cortex) interfere with higher-order cognitive processing (eg, cognitive control) associated with these regions. ${ }^{10,21,22}$ In relation to the altered neuroplasticity part, reduction in gray matter volume in the prefrontal cortex, anterior cingulate cortex, and hippocampus has been found in a pain duration-dependent manner. ${ }^{23,24}$ If pain relief is attained, the volume of gray matter is normalized again. Therefore, it has been suggested that the synapses become inactivated in states of persistent pain. ${ }^{23,24}$ The dysregulated neurochemistry part is built on altered neurochemistry in the brain. ${ }^{25-33}$ In persistent pain, the pain signals are constant, which means that it is unlikely that the brain can repair from these alterations. In regularly recurrent pain, the pain signals are not constant and possibly repair could take place. In acute pain, neuroplastic changes have not been shown and brain activation shows different patterns in acute versus chronic pain. ${ }^{24}$ Brain regions such as the prefrontal cortex, the anterior cingulate cortex, and the secondary somatosensory cortex have been implicated in pain states and play a crucial role in controlled/higher-order cognitive processing involving attention, cognitive control, and certain psychomotor abilities. ${ }^{34,35}$ The aim of this study was to examine how qualitatively different musculoskeletal pain states shape cognitive performance that draws heavily on such brain structures and higher-order mental functions. The current study examined the following specific abilities that tap aspects of sustained attention, cognitive control, and psychomotor ability, respectively: the ability to focus on certain task over prolonged periods of time without being distracted, ${ }^{36}$ the ability to inhibit prepotent response tendencies, ${ }^{37,38}$ and the ability integrate and coordinate perceptual and motor skills. ${ }^{39}$ Given that long-term persistent pain causes the most substantial brain alterations in regions associated with the aforementioned cognitive functions, it was hypothesized that patients with persistent, long-term pain would show more pronounced impairment in cognitive performance than patients with acute or long-term regularly recurrent pain, using pain-free individuals as a benchmark.

\section{Patients and methods}

\section{Study population}

The current study used a cross-sectional comparative study design. The studied groups were created as a result of their preexisting pain characteristics. Inclusion criteria were musculoskeletal pain and Swedish language fluency. Exclusion criteria were diagnosed cognitive impairment, psychiatric diagnoses (except depression as a secondary diagnose), recorded drug or alcohol abuse, motor dysfunction in upper extremities, brain damage, color blindness, and being below 18 years of age.

Patients seeking a physiotherapist at a primary health care facility in southern Sweden for pain in the musculoskeletal system were asked to participate in the study. Pain-free staff from the primary health care center, people accompanying patients to the primary health care center, people from a private company in the southern part of Sweden, and people who had recovered from acute pain were also asked to participate in the study (control group). Individuals who declined to participate ( $\mathrm{N}=11)$ did so due to lack of time or because they did not want to participate in research projects. The current sample consisted of 214 participants ( 72 males, 129 females) aged $18-80$ years (mean $=49$ years, $S D=13$ years). Of these patients, 13 were excluded due to drug or alcohol abuse or due to psychiatric diseases not known to the physiotherapist at the initial meeting. This was performed when the patient record at the primary health care center was searched for exclusion criteria. Every patient was asked about exclusion criteria at the first meeting, and the patient record was searched for exclusion criteria after the session.

Patients were divided into three different pain groups based on the duration and persistence of their pain. The "acute pain group" included patients $(\mathrm{N}=38)$ who had experienced either persistent or regularly recurrent pain for $<3$ months. The "regularly recurrent pain group" included patients ( $\mathrm{N}=58)$ who had experienced regular and recurrent pain at least several times a week and for at least 3 months. The "persistent pain group" included patients $(\mathrm{N}=54)$ who had experienced persistent pain for $>3$ months. The "control group" $(\mathrm{N}=51)$ consisted of pain-free individuals. All participants signed an informed consent form, and the study was approved by the Regional Ethics Review Board (2012/173-31) in Linköping, Sweden.

\section{Cognitive tests}

\section{Sustained attention}

In the current study, we measured sustained attention, ie, the ability to focus on a certain task over prolonged periods of 
time without being distracted. More specifically, attention lapses in the form of moment-to-moment variability of pause time durations (at the millisecond level) were assessed, using the MapCog Spectra (iPad application version). ${ }^{40}$ MapCog Spectra is a relatively new test, which was originally developed for use in attention-deficit hyperactive disorder (ADHD) research. The main reason for using the MapCog Spectra was that we wanted to obtain a sensitive measurement of attention by assessing moment-to-moment fluctuations in attention at the millisecond level. The MapCog Spectra consisted of a presentation of figures (squares, circles, stars, hearts) on an iPad, which were placed in random order in every test session. The patient was asked to mention the color and form of the figures, for every figure, without pausing between the figures. No time measurement was conducted in this test, and the study patients were informed about this. The percentage of the pauses in mentioning the shape and color of the figures (ie, attention lapses) was registered by the iPad application. A percentage above $18 \%$ was considered as deviant. ${ }^{40}$

\section{Cognitive control}

Broadly defined, cognitive control refers to the ability to flexibly switch between different thoughts and actions. ${ }^{38}$ The current research examined one important aspect of cognitive control, namely the ability to inhibit prepotent response tendencies. To this end, the Color Word Test (CWT), also named the Stroop Test, was used. In the crucial part of this test, patients were asked to name the color in which a word was printed, when the text of that word (ie, its meaning) referred to a different color. Extracting the word's semantic meaning was an automatic, habitual process which had to be overcome by a more systematic, less habitual, control process that directed attention to the printed color of the word while ignoring the word's (incongruent) semantic meaning. Thus, the color-naming part in the Stroop task requires more controlled processing compared to the word-reading part that is a more automatic process. ${ }^{37}$ The CWT consists of several parts, which is described elsewhere..$^{41}$ In this study, the parts of naming different colored crosses and naming the color words printed in a different color were used. The time to name the colors in each task was measured (response time), and a difference score was calculated. It has been found that the test-retest reliabilities for the response latencies of naming different colored crosses and conflicting color words are high, where the correlation coefficients are 0.84 and 0.86 , respectively. ${ }^{42}$ Regarding validity, the interference score correlated moderately well with measures of attention and it appeared similar to the ability to resist interference from irrelevant information in the external environment. ${ }^{41}$ No normative value was found for the difference score between naming different colored crosses and conflicting color words.

\section{Psychomotor ability}

With respect to psychomotor ability, we specifically measured visual motor coordination, using the Grooved Peg Board (Model 32025; Lafayette Instrument Company). The unit consisted of 25 holes with randomly positioned slots. Pegs, which had a key along one side, had to be rotated to match the hole before the peg could be inserted. The time for the patient to correctly insert all slots into the holes was measured. Maximal time for the test was 5 minutes. Normative mean value and SD for normal individuals with a mean age of 43.6 years were 69.66 seconds and 19.27, respectively. Test-retest reliability has been shown to be marginal/high with reliability coefficients ranging from 0.67 to 0.86 in normal individuals. With respect to validity, the time measured in performing the Grooved Pegboard showed a modest relation with finger-tapping speed $(-0.35)$. The test showed a moderate association with reaction time $(r=0.31)$ and a high association with processing speed $(r=0.60) .{ }^{41}$

\section{General cognitive impairment}

The Mini-Mental State Examination (MMSE) was a test used to screen for mental impairment ${ }^{41}$ and was frequently used to test for dementia in primary care settings in Sweden. The MMSE score ranged from 0 to 30 , with a cutoff score for severe cognitive pathology at 24 points. Test-retest reliability ranged between 0.8 and 0.95 , and the MMSE showed modest to high correlations with other brief screening tests used to screen for mental impairment. ${ }^{41}$ In this study, the test was used to ascertain that patients had no severe cognitive impairment, such as dementia.

\section{Instruments for measuring pain}

The visual analog scale (VAS) with one end point indicating no pain and the other indicating worst imaginable pain was used..$^{43}$ A plastic VAS from Pfizer, Inc. (New York, NY, USA) was used in this study. Patients were asked to move an indicator line on a movable plastic piece between the end points to indicate their pain. Their estimation resembled a number on the backside of the scale from 0 (no pain) to 10 (worst imaginable pain) with $10 \mathrm{~mm}$ between each number, and this number was used in statistical analysis. Cutoff points for pain-intensity-related interference with functioning have been suggested with mild $(<3.4)$, moderate (3.5-6.4), and severe pain-related interference $(>6.5){ }^{44}$ 


\section{Protocol}

Two different physiotherapists at the Primary Health Care Center conducted a physical examination and diagnosed the patients according to the ICD-10. If the patients fitted into more than one diagnose category, they were placed in the category that resembled the problem for which they were seeking the physiotherapist treatment. The patient was questioned about current medication, pain duration and persistence, painful body areas, diagnoses, and educational level, and this information was recorded. Then, the physiotherapists administered the cognitive tests. Before the study, the physiotherapists received sufficient training in administering the cognitive tests from an experienced psychologist. The patients were classified into one of the abovementioned pain groups according to their anamnestic pain duration and persistence. Educational level was grouped as follows: completed compulsory school, completed upper secondary school, and completed university degree. Medications were taken by some participants in each group. In the group with acute pain ( $\mathrm{N}=9$, taking pain-relieving medication), the pain-relieving medications consisted of paracetamol, nonsteroidal anti-inflammatory drugs (NSAID), and opioids. In the group with regularly recurrent pain $(\mathrm{N}=10$, taking pain-relieving medication), pain-relieving medications were paracetamol, NSAID, and opioids. Medications in the group with persistent pain ( $\mathrm{N}=24$, taking pain-relieving medication) were paracetamol, opioids, NSAID, antidepressant drugs, antiepileptics, sleeping drugs, muscular relaxants, and folic acid analogs. Pain-relieving medication in the acute pain group was used temporarily and in the regularly recurrent pain group sporadically, since these patients did not have constant pain signaling. In the persistent pain group, the pain-relieving medications were used continually, since these patients experienced constant pain signaling.

In terms of procedure, the patients were first comfortably seated in a chair and asked to estimate their pain on the VAS. Next, they completed the following cognitive tests: the MapCog Spectra, the CWT, the Grooved Peg Board, and the MMSE. The MapCog Spectra was always administered first because we wanted to minimize the risk that patients hurried through the test, which might have been the case if it had followed the two response latency tests (the CWT and Grooved Peg Board) that prompted patients to respond quickly. The MMSE was administered last since we did not want to start with the most sensitive test in terms of its purpose (severe cognitive impairment). The order of the CWT and the Grooved Peg Board was alternated between patients.

\section{Statistical analyses}

Statistical analyses were conducted using IBM SPSS statistics for windows (Version 20; IBM Corporation, Armonk, NY, USA). Descriptive analyses were performed to examine the normality of data and homogeneity of variance. Since skewed distribution and heterogeneity of variance were found, data transformation was performed. For data from the Grooved Peg Board, reciprocal transformation was performed. Data from the CWT were log transformed, and data from the MapCog Spectra were square-root transformed. After transformation of data, the distributions approximated normal distributions.

We performed three planned comparisons for the MapCog Spectra, the CWT, and the Grooved Peg Board Test, respectively. Group affiliation was used as the independent variable. When age and educational level were significantly related to the performance on the cognitive tests, these variables were entered as covariates. To rule out that pain-relieving medication would drive group differences on cognitive performance, additional analyses were performed when pain medication was held constant.

For the MMSE, no transformation of data resolved the problem of skewed distribution, and therefore a nonparametric test (Kruskal-Wallis) was performed to test for differences between groups.

To examine the relationship between the VAS score and performance on cognitive tests, partial correlations were performed for the relation between VAS score and the Grooved Peg Board Test and between VAS score and the CWT. In these analyses, education, medication, and age were controlled for, since these variables correlated significantly with the test results. To examine the correlation between VAS score and the performance on the MapCog Spectra Test, Pearson's correlation coefficient was used since age, medication, and education did not show statistically significant correlations with this test result.

\section{Results}

First, as we were primarily interested in how cognitive performance was affected by qualitatively different pain states, we conducted three planned comparisons in which the cognitive performance of patients with acute, regularly recurrent, and persistent pain, respectively, was compared to that of a control group consisting of healthy, pain-free individuals. For each of these comparisons, achieved power to detect a moderate effect size (equivalent to Cohen's $d=0.5$ ) given the sizes of the compared groups was provided. For these calculations, alpha was set at 0.05 , one tailed. Unlike age and education, 
pain-relieving medication could not be used as a covariate when it was significantly related to cognitive performance. The reason for this was that the control group only consisted of individuals who were not in pain and thus were not taking pain-relieving medication, causing such a covariate analysis to collapse. To rule out that medication could explain group differences in cognitive performance when statistically significant group differences emerged, we performed additional analyses only on those participants who did not take any pain-relieving medication, thereby holding pain-relieving medication constant. If group differences remained when pain medication was taken out of the equation, it could not explain our results. Finally, the relationship between pain intensity and performance on the cognitive tests was investigated. Descriptive statistics are found in Tables 1 and 2. Inferential statistics are shown in Figures 1-3.

\section{Sustained attention}

Age and educational level did not have any significant relation to the performance on the MapCog Spectra test and were therefore not treated as covariates in the subsequent analysis. In the first planned comparison, the acute pain group showed no significant difference in performance compared to healthy controls: $F(1,76)=3.050, P=0.085$, Cohen's $d=0.41$, achieved power $=0.69$.

The second planned comparison showed that the group with regularly recurrent pain performed significantly worse compared to healthy controls: $F(1,98)=6.507, P=0.012$, Cohen's $d=0.525$, achieved power $=0.80$.

The third planned comparison revealed that the group with persistent pain performed significantly worse than healthy controls: $F(1,93)=8.201 P=0.005$, Cohen's $d=0.592$, achieved power $=0.78$.

These results remain virtually unchanged when patients taking pain-relieving medication were excluded: acute pain group vs healthy controls $(P<0.213)$, regularly recurrent pain group vs healthy controls $(P<0.012)$, persistent pain group vs. healthy controls $(P<0.005)$. Thus, pain medication could not explain the aforementioned group differences.

A further analysis showed that in the healthy control group, $4 \%$ performed above the deviant limit for attention lapses, compared to $13 \%$ in the acute pain group, $12 \%$ in the

Table I Baseline characteristics of the study population presented in subgroups

\begin{tabular}{|c|c|c|c|c|c|c|c|c|c|c|c|c|}
\hline \multirow[t]{2}{*}{ Characteristics } & \multicolumn{2}{|c|}{$\begin{array}{l}\text { Healthy controls } \\
(n=5 I)\end{array}$} & \multirow[b]{2}{*}{ SD } & \multicolumn{3}{|c|}{$\begin{array}{l}\text { Acute pain } \\
(n=38)\end{array}$} & \multicolumn{3}{|c|}{$\begin{array}{l}\text { Regularly recurrent } \\
\text { pain }(n=58)\end{array}$} & \multicolumn{3}{|c|}{$\begin{array}{l}\text { Persistent pain } \\
(n=54)\end{array}$} \\
\hline & $\begin{array}{l}\text { Number } \\
(\%)\end{array}$ & $\begin{array}{l}\text { Mean } \\
\text { (age range) }\end{array}$ & & $\begin{array}{l}\text { Number } \\
\text { (\%) }\end{array}$ & $\begin{array}{l}\text { Mean } \\
\text { (age range) }\end{array}$ & $\overline{S D}$ & $\begin{array}{l}\text { Number } \\
\text { (\%) }\end{array}$ & Mean & SD & $\begin{array}{l}\text { Number } \\
\text { (\%) }\end{array}$ & Mean & SD \\
\hline Age, years & & $\begin{array}{l}44.9^{a} \\
(18-80)\end{array}$ & 12.8 & & $\begin{array}{l}46.9^{a} \\
(23-73)\end{array}$ & 13.7 & & $\begin{array}{l}49.9^{a} \\
(22-76)\end{array}$ & 13.0 & & $\begin{array}{l}51.6^{\mathrm{a}} \\
(21-80)\end{array}$ & 13.0 \\
\hline Gender (M/F) & $\begin{array}{l}17 / 34 \\
(33 / 67)\end{array}$ & & & $\begin{array}{l}16 / 22 \\
(42 / 58)\end{array}$ & & & $\begin{array}{l}28 / 30 \\
(48 / 52)\end{array}$ & & & $\begin{array}{l}11 / 43 \\
(20 / 80)\end{array}$ & & \\
\hline \multicolumn{13}{|l|}{ Education } \\
\hline Compulsory school & $4(8)$ & & & $7(18)$ & & & $16(28)$ & & & $22(4 I)$ & & \\
\hline $\begin{array}{l}\text { Upper secondary } \\
\text { school }\end{array}$ & $28(55)$ & & & $22(58)$ & & & $31(54)$ & & & $25(46)$ & & \\
\hline University degree & $19(37)$ & & & $9(24)$ & & & $11(18)$ & & & $7(13)$ & & \\
\hline VAS score & & $0.0^{\mathrm{b}}$ & 0.0 & & $2.9^{c}$ & 2.5 & & $2.6^{\mathrm{cd}}$ & 2.2 & & $4.7^{\mathrm{e}}$ & 2.4 \\
\hline Pain duration, days & & 0.0 & 0.0 & & 18 & 16 & & 3,224 & 4,430 & & 4,018 & 3,588 \\
\hline $\begin{array}{l}\text { Pain in more than } \\
\text { one body region }\end{array}$ & $0(0)$ & & & $2(5)$ & & & $25(43)$ & & & $35(65)$ & & \\
\hline Pain-relieving medication & $0(0)$ & & & $9(24)$ & & & $10(17)$ & & & $22(4 I)$ & & \\
\hline
\end{tabular}

Notes: Mean and SD in all groups of age, VAS score, and pain duration are given. Gender distribution in all groups is given. Actual number of participants and frequency of pain in more than one body region in all groups, actual number of participants and frequency of educational level in all groups, and actual number of participants and frequency of intake of pain-relieving medication in all groups at the test session are given. ${ }^{a}$ Nonsignificant. Mean values with different superscripts (b-e) show a significant difference (one-way ANOVA with Bonferroni post hoc, $P<0.05$ ); this means, for example, that ${ }^{b}$ is different from ${ }^{c},{ }^{c d}$ and ${ }^{e}$, but ${ }^{c}$ is not different from ${ }^{c d}$.

Abbreviation: VAS, visual analog scale; SD, standard deviation; $M$, male; F, female.

Table 2 Number of participants with diagnosis according to ICD-10 for every pain group

\begin{tabular}{llllllllll}
\hline Diagnosis & M545 & M544 & M542 & M754 & M797 & M17 & M16 & G448 & Other \\
\hline Acute pain ( $n=54)$ & 17 & 4 & 6 & 6 & 0 & 1 & 0 & 0 & 4 \\
Regularly recurrent pain $(n=58)$ & 12 & 7 & 17 & 12 & 0 & 5 & 0 & 0 & 5 \\
Persistent pain $(n=54)$ & 13 & 7 & 8 & 7 & 9 & 5 & 1 & 2 & 2 \\
\hline
\end{tabular}

Notes: M545, lumbago; M544, lumbago with radiation to lower extremities; M542, cervicalgia; M754, impingement syndrome; M797, fibromyalgia; MI7, arthrosis in knee; MI6, arthrosis in hip; G448, headache syndrome. 


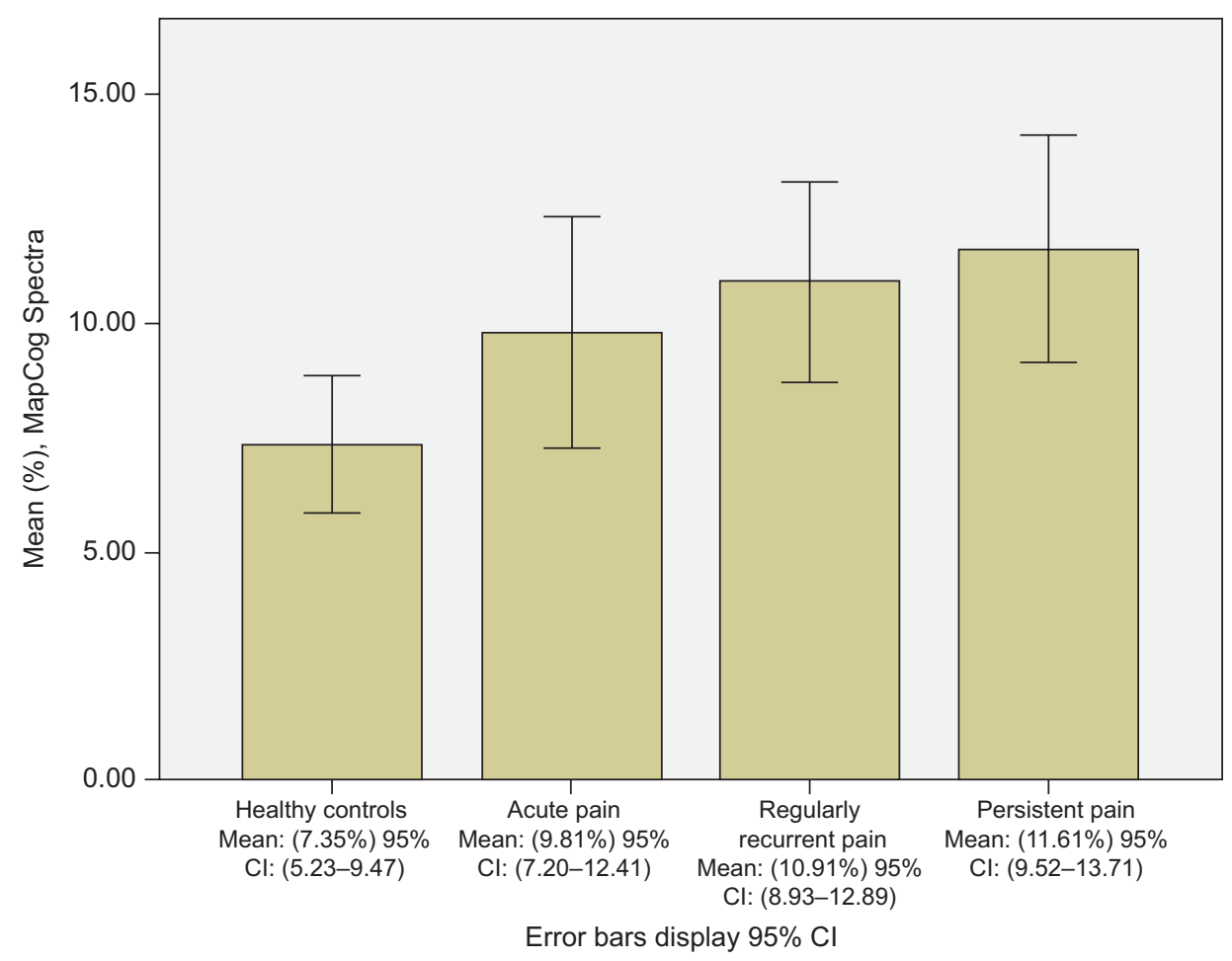

Figure I Performance of the MapCog Spectra (mean and 95\% Cl).

Notes: Performance in percentage of attention lapses presented as mean values for healthy controls, the acute pain group, the regularly recurrent pain group, and the persistent pain group. A higher score indicates worse performance on the test. One-way ANOVAs between subjects revealed that the regularly recurrent pain group and the persistent pain group showed significantly slower performance $(P<0.012$ and $P<0.005$, respectively) compared to healthy controls.

Abbreviation: ANOVA, analysis of variance.

regularly recurrent pain group and $17 \%$ in the group with persistent pain.

\section{Cognitive control}

Age and educational level were significantly related to the performance on the CWT. When controlling for these covariates, none of the three planned comparisons that compared each pain group with healthy controls reached statistical significance: acute pain group vs healthy controls: $F(1,84)=1.300, P=0.257$, Cohen's $d=0.30$, achieved power $=0.74$; regularly recurrent pain group vs healthy controls: $F(1,102)=0.398, P=0.530$, Cohen's $d=0.332$, achieved power $=0.82$; and persistent pain group vs healthy controls: $F(1,98)=1.498, P=0.224$, Cohen's $d=0.512$, achieved power $=0.81$.

\section{Psychomotor ability}

Age and education level were significantly related to the performance on the Grooved Peg Board Test. When controlling for these covariates, the first planned comparison showed that the acute pain group performed significantly worse than healthy controls: $F(1,85)=9.119, P=0.003$, Cohen's $d=0.392$, achieved power $=0.75$.
The second planned comparison showed no significant difference in performance for the group with regularly recurrent pain compared to healthy controls: $F(1,102)=1,811$ $P=0.181$, Cohen's $d=0.39$, achieved power $=0.82$.

The third planned comparison showed that the persistent pain group performed significantly worse than healthy controls: $F(1,101)=15,498, P=0.000$, Cohen's $d=0.78$, achieved power $=0.82$.

These results remained virtually unchanged when patients taking pain-relieving medication were excluded: acute pain group vs healthy controls $(P<0.012)$, regularly recurrent pain group vs healthy controls $(P<0.195)$, persistent pain group vs healthy controls $(P<0.008)$. Thus, pain medication could not explain the aforementioned group differences.

A further analysis showed that $20 \%$ of healthy controls performed above the normative mean value compared to $39 \%$ in the acute pain group, $31 \%$ in the regularly recurrent pain group, and $57 \%$ in the persistent pain group.

\section{Mini-Mental State Examination}

There was no significant difference among groups on the MMSE. No group showed severe cognitive impairment (<24 points). 


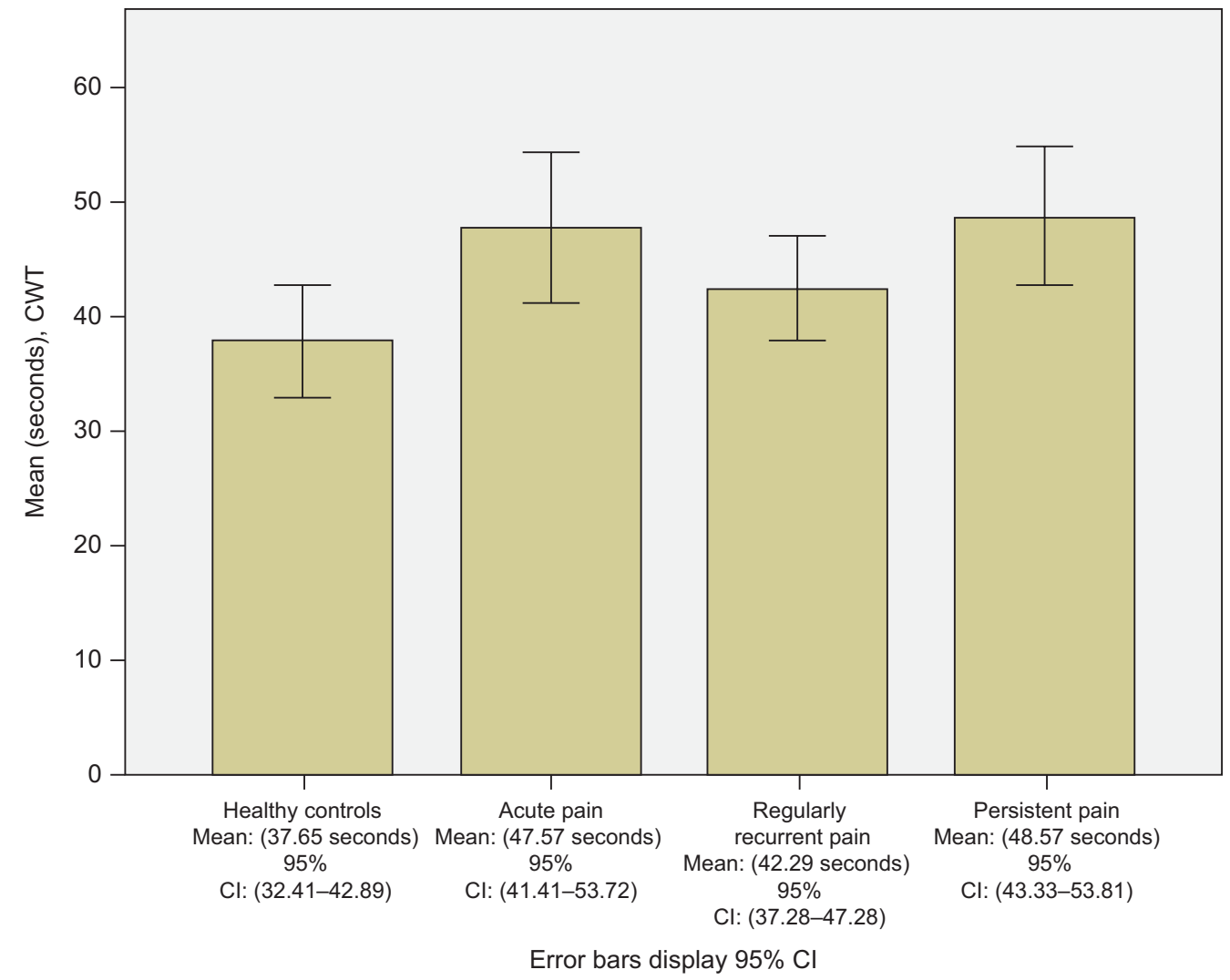

Figure 2 Performance of the CWT (mean and $95 \% \mathrm{Cl}$ ).

Notes: Performance on the CWT (difference score) presented as mean values for healthy controls, the acute pain group, the regularly recurrent pain group, and the persistent pain group. A higher score indicates worse performance on the test. One-way ANCOVAs between subjects (controlling for age and education) showed that healthy controls did not differ significantly from any of the three pain groups.

Abbreviations: ANCOVA, analysis of covariance; CWT, Color Word Test.

\section{Correlation between pain intensity and performance on cognitive tests}

As all participants in the pain-free control group had a VAS score of zero, correlations between VAS and test performance were based on the aggregated data for the three pain groups. There were no significant relationships between VAS score and cognitive performance in any of the three cognitive tests. More specifically, the partial correlations (controlling for age, education, and medication) between the VAS score and the CWT and between the VAS score and the Grooved Pegboard Test were $r=-0.026, P<0.772$ and $r=-0.092, P<0.307$, respectively. The correlation between VAS score and the MapCog Spectra Test was $r=-0.009, P<0.919$.

\section{Discussion}

We hypothesized that patients with persistent, long-term pain would show more pronounced impairment in cognitive performance than patients with acute or long-term regularly recurrent pain. Indeed, we found that the persistent pain group evidenced significantly lower levels of both sustained attention and visual motor coordination performance when compared to a comparison group consisting of pain-free individuals. These differences were moderate in magnitude according to the conventional effect size standards $(d>0.5)$. Although persistent musculoskeletal pain seems to impair performance on a wider range of cognitive tasks and to a greater extent, as determined by the observed effect sizes, than does acute or long-term regularly recurrent pain, we also found evidence of some cognitive impairment among patients with acute pain and among patients with long-term regularly recurrent pain. Specifically, patients with longterm regularly recurrent pain appear to perform worse with respect to sustained attention (moderate effect size) and patients with acute pain seem to perform worse with respect to visual motor coordination (small effect size) relative to pain-free controls. The results are consistent with our reasoning that long-term persistent pain should have the largest impact on brain regions responsible for higher-order cognitive processing. The results also give support to the limited resource theory as some, albeit less, pronounced impairment also occurs in patients with acute and long-term regularly recurrent pain. 


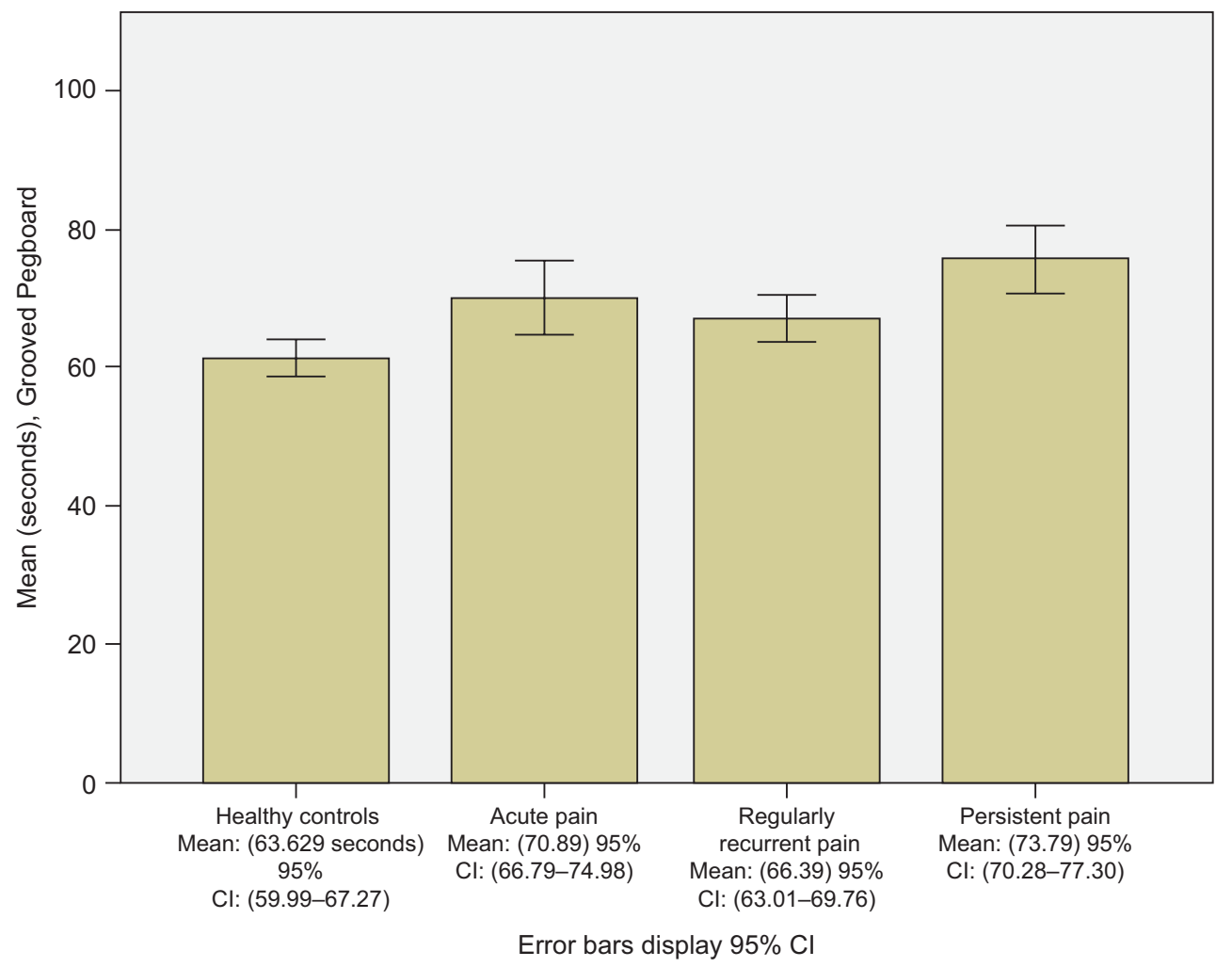

Figure 3 Performance of the Grooved Pegboard (mean and 95\% $\mathrm{Cl}$ ).

Notes: Performance in seconds of the Grooved Pegboard presented as mean values for healthy controls, the acute pain group, the regularly recurrent pain group, and the persistent pain group. A higher score indicates worse performance on the test. One-way ANCOVAs between subjects (controlling for age and education) revealed that the acute pain group and the persistent pain group showed significantly slower performance $(P<0.003 ; P<0.000$, respectively) compared to healthy controls.

Abbreviation: ANCOVA, analysis of covariance.

Our results further suggest that pain intensity matters little for cognitive performance on the measured tasks as no significant correlations emerged between the degree of reported pain and cognitive performance. Although our data are more consistent with the view that persistence rather than intensity matters for cognitive performance, pain intensity is more likely to matter more for cognitive performance in more extreme populations who experience higher levels of pain than that of the current study. Needless to say, if pain intensity becomes too extreme, people may not be able to take part in cognitive testing to begin with.

\section{Sustained attention}

When measuring rapid changes in attention, a significantly decreased ability in attention (moderate effect size) was found in the persistent pain group and in the regularly recurrent pain group compared to pain-free controls. Since MapCog Spectra is a newly developed test used in ADHD research, there are no previous published studies on pain which have used this test. Our finding is consistent with the theory of pain and attention disruption, which proposes that individuals with pain have a disruption in attention, since they must switch between pain signals and other attention engaging actions in the environment. ${ }^{13}$ In a health care environment, this could be important when informing patients about their treatment and prognosis. In patients with impaired attentional ability, the same information should be presented several times and in different ways: both oral and written information could be presented.

\section{Cognitive control}

No significant differences in performance between any of the groups were found in the test measuring cognitive control. This result is not in accordance with earlier studies of cognitive control, where an effect of impaired performance has been found. ${ }^{9,11-13}$ Using the Stroop Test, it was found that only patients with high-intensity chronic pain (mean VAS score 4.7) showed decreased performance compared to healthy controls. ${ }^{11}$ Another study, measuring an interference task, found that patients with high-intensity chronic pain had significant deficits in performance, while patients with low-intensity chronic pain showed no significant impairment in the task (the cutoff point between high- and low-intensity pain in VAS score was 3.96). ${ }^{12}$ In addition, another study reported a significant correlation between pain ratings and performance of the Stroop interference task in patients in a small sample with different pain diagnoses.$^{45}$ From 
this, it could be suggested that the intensity of the attentiondisturbing pain stimuli matters for the process of cognitive control. However, in the Stroop interference task mentioned earlier, the numerical pain ratings for patients with high-intensity chronic pain were similar to the VAS pain ratings in the group with persistent pain in our study (a mean of 4.7 in both studies), and therefore pain intensity does not seem to be a likely explanation for the difference in results between these studies. Furthermore, the correlation in our study between VAS score and cognitive performance was nonexistent. In this context, it does not seem probable that pain intensity affected cognitive performance in our study. However, it has been debated that the inconsistency in results between different studies could be due to the heterogeneity in sampling, since subtypes of different chronic pain states and pain conditions are complex and often inadequately described in studies. ${ }^{17}$

\section{Psychomotor ability}

The significantly decreased performance in psychomotor ability for patients with long-term persistent pain is consistent with an earlier study, where significantly impaired performance was found in older individuals with chronic, but not necessarily persistent pain, compared to pain-free controls. ${ }^{46}$ Other studies, suggesting that long-term persistent pain is responsible for functional and structural changes in the brain, ${ }^{23,24,47,48}$ could be important when interpreting the results. For example, it has been shown that the longer the pain duration, the greater the decreases in gray matter. Nowadays, it is not believed that the cause of this decrease is apoptosis, but instead reduced dendritic or synaptic density. It has also been suggested that the pattern and number of links between different brain regions shift in individuals with persistent pain. If the structure of networks in the brain is changed in persistent pain states, it could be suggested that the efficiency in brain function is decreased. ${ }^{47}$ This may explain the slower performance in a psychomotor task in patients with long-term persistent pain compared to healthy controls. In relation to the significant, yet small, performance impairment with respect to the psychomotor task among patients with acute pain, this could be understood in the context of the limited resource theory, where it has been suggested that pain signals from the spinal cord to higher brain regions interfere with higher-order cognitive processing. ${ }^{10}$

\section{Severe cognitive impairment}

With respect to MMSE, there were no significant group differences. This is important because it shows that the groups do not differ with respect to severe cognitive deficits, such as dementia. The result is consistent with an earlier study examining cognitive impairment in patients with neuropathic pain and muscular pain. ${ }^{49}$

\section{Strength and limitations}

A limitation of the current study is that no measures of depression as a secondary diagnosis were controlled for. Earlier studies on the subject have been inconclusive. It has been concluded that comorbid depression, anxiety, and sleep disturbances were unrelated to cognitive test performance in patients with fibromyalgia syndrome, ${ }^{50}$ and some other studies found no relation between depression or anxiety on executive function in patients with chronic pain. ${ }^{14,20}$ However, there are other studies that found that depression alters performance in decision-making tasks, ${ }^{22}$ and in a group of cognitively impaired fibromyalgia patients the impairment was diminished when covariates such as depression, anxiety, average hours of sleep per night, and number of nighttime awakenings were controlled for. ${ }^{16}$ It has also been suggested that depression in patients with chronic pain alone could account for significant impairment in cognitive performance. ${ }^{51}$ This is interesting because there are factors coexisting with chronic pain, such as poor sleep, depression, and anxiety which can also affect cognitive performance. Alterations in brain activation and interference with attention processes after sleep deprivation have been found in earlier studies ${ }^{52,53}$ and therefore measures of disturbed sleep should be taken into account in future research.

In our study, we were able to rule out that medication explained the uncovered group differences on cognitive performance. This is consistent with earlier studies that have examined the effect of the use of selective opioids or psychotropic drug treatment on cognitive performance in patients with chronic pain. ${ }^{20,54-57}$

\section{Conclusion}

The results of the current study suggested that persistent musculoskeletal pain impaired performance on a wider range of cognitive tasks and to a somewhat greater extent than did acute or long-term regularly recurrent pain, using pain-free individuals as a benchmark. However, we found some evidence of impairment in psychomotor ability among patients with acute pain and some impairment in sustained attention among patients with long-term regularly recurrent pain. In addition, we found that pain intensity seemed to matter little for cognitive performance on the current tasks, at least in the current pain population. Our results were more consistent with the view that persistence and duration, rather than pain intensity, contribute to impaired cognitive function. 


\section{Acknowledgments}

We thank Hälsoringen, Neron HSU AB, Osby, Sweden, for data collection resources.

\section{Disclosure}

The authors report no conflicts of interest in this work.

\section{References}

1. Carr DB, Eisenberg E, Friction JR, et al. Editorial Board: Cognitive Impairment in Chronic Pain. Vol. XV. IASP: Pain Clinical Updates . Washington; 2007:4.

2. Oosterman JM, Derksen LC, van Wijk AJM, Kessels RPC, Veldhuijzen DS. Executive and attentional functions in chronic pain: does performance decrease with increasing task load? Pain Res Manage. 2012;17(3):159-165.

3. Wolrich J, Poots AJ, Kuehler MN, Rice ASC, Rahman A, Bantel C. Is number sense impaired in chronic pain patients? Br J Anaesth. 2014;113(6):1024-1031.

4. Melkumova KA, Podchufarova EV, Yakhno NN. Characteristics of cognitive functions in patients with chronic spinal pain. Neurosci Behav Physiol. 2011;41:42-46.

5. Berryman C, Stanton TR, Bowering KJ, Tabor A, McFarlane A, Moseley GL. Evidence for working memory deficits in chronic pain: a systemativ review and meta-analysis. Pain. 2013;154(8):1181-1196.

6. Berryman C, Stanton TR, Bowering KJ, Tabor A, McFarlane A, Moseley GL. Do people with chronic pain have impaired executive function? A meta-analytical review. Clin Psychol Rev. 2014;34(7):563-579.

7. Attridge N, Noonan D, Eccleston C, Keogh E. The disruptive effects of pain on n-back task performance in a large general population sample. Pain. 2015;156(10):1885-1891.

8. Duschek S, Werner NS, Winkelmann A, Wankner S. Implicit memory function in fibromyalgia syndrome. Behav Med. 2013;39(1):11-16.

9. Moore DJ, Keogh D, Eccleston C. The interruptive effect of pain on attention. Q J Exp Psychol. 2012;65(3):565-586.

10. Moriarty O, McGuire BE, Finn DP. The effect of pain on cognitive function: a review of clinical and preclinical research. Prog Neurobiol. 2011;93(3):385-404.

11. Grisart JM, Plaghki LH. Impaired selective attention in chronic pain patients. Eur J Pain. 1999;3(4):325-333.

12. Eccleston $\mathrm{C}$. Chronic pain and distraction: an experimental investigation into the role of sustained and shifting attention in the processing of chronic persistent pain. Behav Res Ther. 1995;33(4):391-405.

13. Eccleston $C$, Crombez G. Pain demands attention: a cognitive-affective model of the interruptive function of pain. Psychol Bull. 1999;125(3): 356-366.

14. Dick B, Eccleston C, Crombez G. Attentional functioning in fibromyalgia, rheumatoid arthritis and musculoskeletal pain patients. Arthritis Rheum. 2002;47(6):639-644.

15. Dick BD, Rashiq S. Disruption of attention and working memory traces in individuals with chronic pain. Anaesth Analg. 2007;104(5):1223-1229.

16. Dick BD, Verrier MJ, Harker KT, Rashiq S. Disruption of cognitive function in fibromyalgia syndrome. Pain. 2008;139(3):610-616.

17. Liu X, Li L, Tang F, Wu S, Hu Y. Memory impairment in chronic pain patients and the related neuropsychological mechanisms: a review. Acta Neuropsychiatr. 2014;26(4):195-201.

18. Keogh D, Moore DJ, Duggan GB, Payne SJ, Eccleston C. The disruptive effects of pain on complex cognitive performance and executive control. PLoS One. 2013;8(12):e83272.

19. Crombez G, Eccleston C, Baeyens F, Eeleen P. The disruptive nature of pain. An experimental investigation. Behav Res Ther. 1996;34(11-12):911-918.

20. Cuevaz-Toro AM, Lopez-Torrecillas F, Diaz-Batanero C, Perez-Marfil MN. Neuropsychological function, anxiety, depression and pain impact in fibromyalgia patients. Span J Psychol. 2014;17:1-9.
21. Apkarian AV, Bushnell MC, Treede R, Zubieta J. Human brain mechanisms of pain perception and regulation in health and disease. Eur $J$ Pain. 2005;9(4):463-484.

22. Neugebauer V, Galhardo V, Maione S, Mackey SC. Forebrain pain mechanisms. Brain Res Rev. 2009;60(1):226-242.

23. Kuchinad A, Schweinhardt P, Seminowicz DA, Wood PB, Chizh BA, Bushnell MC. Accelerated brain gray matter loss in fibromyalgia patients: premature aging of the brain? J Neurosci. 2007;27(15):4004-4007.

24. Apkarian AV, Bushnell MC, Schweinhardt P. Representation of pain in the brain. In: McMahon SB, Koltzenburg M, Tracey I, Turk DC, editors. Wall and Melzacks Textbook of Pain. Philadelphia: Elsevier Saunders; 2013:111-129.

25. Finn DP, Jhaveri MD, Beckett SR, et al. Behavioral, central monoaminergic and hypothalamo-pituitary-adrenal axis correlates of fearconditioned analgesia in rats. Neuroscience. 2006;138(4):1309-1317.

26. Omote K, Kawamata T, Kawamata M, Namiki A. Formalin-induced nociception activates a monoaminergic descending inhibitory system. Brain Res. 1998;814(1-2):194-198.

27. Wood PB, Schweinhardt P, Jaeger E, et al. Fibromyalgia patients show an abnormal dopamine response to pain. Eur J Neurosci. 2007; 25(12):3576-3582.

28. Wood PB. Role of central dopamine in pain and analgesia. Expert Rev Neurother. 2008;8(5):781-797.

29. Wood PB, Glabus MF, Simpson R, Patterson JC 2nd. Changes in gray matter density in fibromyalgia: correlation with dopamine metabolism. J Pain. 2009;10(6):609-618.

30. Wood PB, Holman AJ. An elephant among us: the role of dopamine in the pathophysiology of fibromyalgia. J Rheumatol. 2009;36(2):221-224.

31. Inoue K, Tsuda M. Microglia and neuropathic pain. Glia. 2009;57(14): 1469-1479.

32. McMahon SB, Cafferty WB, Marchand F. Immune and glial cell factors as pain mediators and modulators. Exp Neurol. 2005;192(2):444-462.

33. Miller RJ, Jung H, Bhangoo SK, White FA. Cytokine and chemokine regulation of sensory neuron function. Handb Exp Pharmacol. 2009; 194:417-449.

34. Chen TL, Babiloni C, Ferreti A, et al. Human secondary somatosensory cortex is involved in the processing of somatosensory rare stimuli: an fMRI study. Neuroimage. 2008;40(4):1765-1771.

35. Babiloni C, Vecchio F, Bares M, et al. Functional coupling between anterior prefrontal cortex (BA10) and hand muscle contraction during intentional and imitative motor acts. Neuroimage. 2008;39(3): 1314-1323.

36. Matlin MW. Cognition. 6th ed. Hoboken, New Jersey: Wiley; 2005.

37. Botvinik MM, Cohen JD. The computational and neural basis of cognitive control: charted territory and new frontiers. Cogn Sci. 2014;38(6): $1-37$.

38. Dreisbach G. Mechanisms of cognitive control: the functional role of task rules. Curr Dir Psychol Sci. 2012;21:227-231.

39. Wang L, Krasich K, Bel-Bahar T, Hughes L, Mitroff SR, Appelbaum G. Mapping the structure of perceptual and visual-motor abilities in healthy young adults. Acta Psychol. 2015;157:74-84.

40. Warkentin S, Carlsson R, Mohammed AKH, Terjestam Y, Persson BA. Linking attentional lapses to perceptual fragmentation: Preliminary findings with the MapCog Spectra (MCS). Poster session presented at: 5 th Conference of the European Societies of Neuropsychology and the 12th Nordic Meeting in Neuropsychology; September 2015; Tampere, Finland.

41. Strauss E, Sherman EMS, Spreen O. A Compendium of Neuropsychological Tests. 3rd ed. Oxford: University Press; 2006.

42. Siegrist M. Reliability of the stroop test with single-stimulus presentation. Percept Mot Skills. 1995;81(3 pt 2):1295-1298.

43. Gracely RH. Studies of pain in human subjects. In: McMahon SB, Koltzenburg M, Tracey I, Turk DC, editors. Wall and Melzacks Textbook of Pain. Philadelphia: Elsevier Saunders; 2013:283-300.

44. Boonstra AM, Shiphorst Preuper HR, Balk GA, Stewart RE. Cut-off points for mild, moderate, and severe pain on the visual analogue scale for pain in patients with chronic musculoskeletal pain. Pain. 2014;155(12):2545-2550 
45. Dick B, Eccleston C, Crombez G. Attentional functioning in fibromyalgia, rheumatoid arthritis and musculoskeletal pain patients. Arthritis Rheum. 2002;47(6):639-644.

46. Weiner DK, Rudy TE, Morrow L, Slaboda J, Lieber S. The relationship between pain, neuropsychological performance, and physical function in community-dwelling older adults with chronic low back pain. Pain Med. 2006;7(1):60-70.

47. Farmer MA, Baliki MN, Apkarian AV. A dynamic network perspective of chronic pain. Neurosci Lett. 2012;520(2):197-203.

48. Grachev ID, Fredrickson BE, Apkarian AV. Abnormal brain chemistry in chronic back pain: an in vivo proton magnetic resonance spectroscopy study. Pain. 2000;89(1):7-18.

49. Povedano M, Gascon J, Galvez R, Ruiz M, Rejas J. Cognitive function impairment in patients with neuropathic pain under standard conditions of care. J Pain Symptom Manage. 2007;33(1):78-89.

50. Reyes del Paso GA, Pulgar A, Duschek S, Garrido S. Cognitive impairment in fibromyalgia syndrome: the impact of cardiovascular regulation, pain, emotional disorders and medication. Eur J Pain. 2012;16(3):421-429.

51. Brown SC, Glass JM, Park DC. The relationship of pain and depression to cognitive function in rheumatoid arthritis patients. Pain. 2002;96(3):279-284.
52. Elvemo NA, Landro ND, Borchgrevink PC, Håberg AK. A particular effect of sleep, but not pain or depression, on the blood-oxygen-level dependent response during working memory tasks in patients with chronic pain. J Pain Res. 2015;8:335-346.

53. Miro E, Lupian J, Hita E, Martinez MP, Sanchez AI, Buela-Casal G. Attentional deficits in fibromyalgia and its relationship with pain, emotional distress and sleep dysfunction complaints. Psychol Health. 2011;26(6):765-780.

54. Lorenz J, Beck H, Bromm B. Cognitive performance, mood and experimental pain before and during morphine-induced analgesia in patients with chronic non-malignant pain. Pain. 1997;73(3):369-375.

55. O’Neill W, Hanks G, White L, Simpson P, Wesnes K. The cognitive and psychomotor effects of opioid analgesics. I. A randomized controlled trial of single doses of dextropropoxyphene, lorazepam, and placebo in healthy subjects. Eur J Clin Pharmacol. 1995;48(6):447-453.

56. Tassain V, Attal N, Fletcher D, et al. Long term effects of oral sustained release morphine on neuropsychological performance in patients with chronic non-cancer pain. Pain. 2003;104(1-2):389-400.

57. Walker D, Zacny J. Subjective, psychomotor, and analgesic effects of oral codeine and morphine in healthy volunteers. Psychopharmacology (Berl). 1998;140(2):191-201.

\section{Journal of Pain Research}

\section{Publish your work in this journal}

The Journal of Pain Research is an international, peer reviewed, open access, online journal that welcomes laboratory and clinical findings in the fields of pain research and the prevention and management of pain. Original research, reviews, symposium reports, hypothesis formation and commentaries are all considered for publication.

\section{Dovepress}

The manuscript management system is completely online and includes a very quick and fair peer-review system, which is all easy to use. Visit http://www.dovepress.com/testimonials.php to read real quotes from published authors. 